\title{
Novel Semi-Blind Channel Estimation Schemes for Rayleigh Flat Fading MIMO Channels
}

\author{
Jaymin Bhalani $^{1}$, Dharmendra Chauhan ${ }^{1}$, Y. P. Kosta ${ }^{2}$, A. I. Trivedi ${ }^{3}$ \\ ${ }^{1}$ Department of Electronics Engineering, Charotar University of Science and \\ Technology, Changa, India \\ ${ }^{2}$ Marvadi Education Institute, Rajkot, India \\ ${ }^{3}$ Department of Electrical Engineering, Faculty of Technology, M.S.University, Baroda, India \\ E-mail: jaymin188@gmail.com, dharmendrachauhan.ec@ecchanga.ac.in, \\ ypkosta@gmail.com,aitrivedi@yahoo.com \\ Received July 17, 2011; revised August 22, 2011; accepted September 1, 2011
}

\begin{abstract}
In this paper, we propose two novel semi-blind channel estimation techniques based on QR decomposition for Rayleigh flat fading Multiple Input Multiple output (MIMO) channel using various pilot symbols. In the first technique, the flat-fading MIMO channel matrix $\mathrm{H}$ can be decomposed as an upper triangular matrix $\mathrm{R}$ and a unitary rotation matrix $\mathrm{Q}$ as $\mathrm{H}=\mathrm{RQ}$. The matrix $\mathrm{R}$ is estimated blindly from only received data by using orthogonal matrix triangularization based house holder QR decomposition, while the optimum rotation matrix Q is estimated exclusively from pilot based Orthogonal Pilot Maximum Likelihood Estimator (OPML) algorithm. In the second technique, joint semi-blind channel and data estimation is performed using QR decomposition based Least Square (LS) algorithm. Simulations have taken under 4-PSK data modulation scheme for two transmitters and six receiver antennas using various training symbols. Finally, these two new techniques compare with Whitening Rotation (WR) based semi-blind channel estimation technique and results shows that those new techniques achieve very nearby performance with low complexity compare to Whitening rotation based technique. Also first technique with perfect $\mathrm{R}$ outperforms Whitening Rotation based technique.
\end{abstract}

Keywords: Multiple Input Multiple Output, Orthogonal Pilot ML Estimator, QR Decomposition, Semi Blind Channel Estimation

\section{Introduction}

A Multiple Input Multiple Output (MIMO) communication system uses multiple antennas at the transmitter and receiver to achieve numerous advantages. Traditionally, antenna arrays have been used at the transmitter and the receiver to achieve array gain, which increases the output SNR of the system. More recently, ways of using multiple antennas has been discovered to achieve diversity and multiplexing gain by exploiting the once negative effect of multipath. Under suitable conditions, i.e. a scatter rich environment, the channel paths between the different transmit and receive antennas can be treated as independent channels due to the multipath effects caused by the scatterers. Channel state information (CSI) provides key information for the operation of MIMO wireless communication systems and hence need to be esti- mated accurately. Many channel estimation algorithms have been developed in recent years. In the literature [1-4], MIMO channel estimation methods can be classified into three classes: training based, blind and semiblind. For pure training based scheme, a long training is necessary in order to obtain a reliable channel estimate, which considerably reduces system bandwidth efficiency. In Blind methods, no training symbols are used and channel state information is acquired by relaying on the received Signal statistics [5-8], which achieves high system throughput requiring high computational complexity. Semi-blind channel estimation approaches as a combination of the two aforementioned procedures [9-11], with few training symbols along with blind statistical information, such techniques can solve the convergence problems and high complexity associated with blind estimators. Extensive work has been done later by Slock et 
al. [12,13] where several semi-blind techniques have been reported. Whitening Rotation (WR) based semi blind technique with Orthogonal Pilot Maximum Likelihood (OPML) [14-17] has shown very good performance compare to other sub-optimal techniques and training based channel estimation techniques. WR technique is less computationally complex compare to other matrix inversion based channel estimation techniques, still there is computational complexity related to SVD calculation in WR based technique so there is requirement to proposed matrix decomposition based semi-blind channel estimation technique which is less complex and have good performance [18-23]. Here we have proposed two novel semi-blind channel estimation techniques. In the first technique, MIMO channel matrix $\mathrm{H}$ is decomposed as $H=R Q$, where $\mathrm{R}$ is upper triangular matrix, which is estimated blindly from only received data using matrix triangularization based house holder QR decomposition while $\mathrm{Q}$ is estimated using training symbols based Orthogonal Pilot Maximum Likelihood algorithm. Second proposed technique is based on joint semi-blind channel and data estimation. The main steps of this novel method are as follows:

Step 1: Initial training based channel estimation is performed using QR decomposition based Least Square (LS) algorithm with the help of training input and training output.

Step 2: Given channel knowledge (estimate), perform data estimation.

Step 3: Given data estimation and received Output, perform blind channel estimation using Same method.

These two new proposed techniques shows near by performance compare to WR based techniques with low computational cost and first technique with perfect knowledge of $\mathrm{R}$ shows better result. The remainder of this paper is organized as follows. The second section describes the system model. The estimation algorithms with proposed techniques are presented in Section 3, simulation results and discussion provides in Section 4 and Section 5 concludes this paper.

\section{System Model}

Consider a flat fading MIMO channel matrix $H \in c^{r \times t}$ where $t$ is the number of transmit antennas and $r$ is the number of receive antennas in the system, and each $h_{i j}$ represents the flat-fading channel coefficient between the $i^{\text {th }}$ receiver and $j^{\text {th }}$ transmitter. Denoting the complex received data by $Y \in c^{r \times 1}$, the equivalent base-band system can be modelled as

$$
Y(k)=H X(k)+\eta(k)
$$

where

$$
H=\left[\begin{array}{cccc}
h_{11} & h_{12} & \cdots & h_{1 t} \\
h_{21} & h_{22} & \cdots & h_{2 t} \\
\vdots & \vdots & \ddots & \vdots \\
h_{r 1} & h_{r 2} & \cdots & h_{r t}
\end{array}\right]
$$

$k$ represents the time instant, $X \in c^{t \times 1}$ is the complex transmitted symbol vector. $\eta$ is additive white Gaussian noise such that $E\{\eta(k) \eta(l)\}=\delta(k, l) \sigma_{n}^{2} I$ where $\delta(k, l)=1$ if $k=1$ and 0 otherwise. Also, the sources are assumed to be spatially and temporally independent with identical source power $\sigma_{s}^{2}$ i.e. $E\{X(k) X(l)\}=$ $\delta(k, l) \sigma_{s}^{2} I$. The signal to noise ratio (SNR) of operation is defined as $\operatorname{SNR} \frac{\sigma_{s}^{2}}{\sigma_{n}^{2}}$ Assume that the channel has been used for a total of $\mathrm{N}$ symbol transmissions. Out of these $\mathrm{N}$ transmissions, the first $\mathrm{L}$ symbols are known training symbols and reaming $\mathrm{N}-\mathrm{L}$ is blind data symbols.

\section{Channel Estimation Techniques}

\subsection{Whitening Rotation Based Semi-Blind Channel Estimation}

Now consider a MIMO channel $H \in c^{r \times t}$ which has at least as many receive antennas as transmit antennas i.e. $r \geq t$. Then, the channel matrix $H$ can be decomposed as $H=W Q^{H}$ where $W \in c^{r \times t}$ is also known as the whitening matrix and $Q \in c^{t \times t}$, termed as the rotation matrix, that is unitary i.e. $Q^{H} Q=Q Q^{H}=I$. As shown in [14], the matrix $W$ can be estimated from the received data alone. We therefore employ the pilot information to exclusively estimate the rotation matrix $Q$. This semi-blind estimation procedure is termed as a Whitening-Rotation (WR) scheme. Let the Singular Value Decomposition (SVD) of $\mathrm{H}$ be given as $P \sum Q^{H}$. A possible choice for $W$ is given by $W=P \sum$ and we assume this specific choice in the rest of the work. We present next a list of potential assumptions which are employed as appropriate in subsequent parts of the work.

Assumption A. $W \in c^{r \times t}$ is perfectly known at the output.

Assumption B. $X \in c^{t \times L}$ is orthogonal i.e. $X X^{H}=\sigma_{s}^{2} L I_{t \times t}$

Assumption A is reasonable if we assume the transmission of a long data stream $(\mathrm{N} \rightarrow \infty)$ from which $W$ can be estimated with considerable accuracy and Assumption B can be easily achieved by using an integer orthogonal structure such as the Hadamard matrix.

$\hat{Q}: c^{r \times L} \rightarrow S$, where $\hat{Q}$ the constrained ML estimator of is $Q$ and $S$ is the manifold of unitary matrices, is obtained by minimizing the likelihood 


$$
\left\|Y-W Q^{H} X\right\|^{2} \text { Such that } Q Q^{H}=I
$$

$M=W^{H} Y X^{H}$. We then have the following result for the constrained estimation of $Q$. Under both assumption $\hat{Q}$ the constrained OPML estimate of $Q$ that minimizes the cost function in (2) is given by

$$
\hat{Q}=V_{M} U_{M}^{H} \text { where, } U_{M} \Sigma_{M} V_{M}^{H}=\operatorname{SVD}(M)
$$

This technique has been proposed and proved in [14] since this procedure employs assumption B. (orthogonal pilot), which termed as the OPML estimator. The above expression (3) thus yields a closed form expression for the computation of $\hat{Q}$, the ML estimate of $Q$. The channel matrix $\mathrm{H}$ is then estimated as $\hat{H}=W \hat{Q}_{H}$.

\subsection{House Holder QR-OPML (Proposed Method-I)}

A novel algorithm is proposed and studied for QR decomposition-based scheme in the context of semi-blind Multi-Input Multi-Output (MIMO) channel estimation. Specifically, the flat-fading MIMO channel matrix $H$ can be decomposed as a upper triangular matrix $R$ and a unitary rotation matrix $Q$. The matrix $R$ is estimated blindly from only received data by using orthogonal matrix triangularization based house holder QR decomposition while the optimum rotation matrix $Q$ is estimated exclusively from training samples based on OPML (Orthogonal Pilot ML Estimator) technique.

The output $Y$ can be written as

$$
Y=H X+N
$$

Output covariance matrix can be described as

$$
\begin{gathered}
R_{Y Y}=H X(H X)^{H}+\sigma_{n}^{2} I \\
R_{Y Y}=H H^{H}+\sigma_{n}^{2} I
\end{gathered}
$$

Now apply householder transformation to received output covariance $R_{Y Y}$. In the householder approach, a series of reflection matrix is applied to matrix $R_{Y Y}$ column by column to annihilate the lower triangular elements. The reflection transformations are ortho-normal matrices that can be written as

$$
A=\left(I+\beta V V^{H}\right)
$$

where $V$ is the Householder vector and $\beta=-2\|V\|_{2}^{2}$.

For the matrix $R_{Y Y}$ to annihilate the lower elements of the $K$-th column the $A_{k}$ is Constructed as follows:

1) Let $V$ equal the $k$-th column of $R_{Y Y}$ $0,0]^{T}$

2) Update $\mathrm{V}$ by $V=R_{Y Y}+\left\|R_{Y Y}\right\|_{2} \varphi$ where $\psi=[1,0,0$,

3) Determine $\beta$ equal to $\beta=-2\|V\|_{2}^{2}$

4) $A_{k}$ is calculated as $A=\left(I+\beta V V^{H}\right)$
The from the above steps are pre-multiplied by $R_{Y Y}$ sequentially as follows

$$
A_{n}, \cdots, A_{1} R_{Y Y}=\left[\begin{array}{c}
R \\
0
\end{array}\right]
$$

where, $R$ is an $n \times n$ upper triangular matrix, 0 is a null matrix, and the sequences of reflection matrices form the complex transpose of the orthogonal matrix $Q^{H}$, where $Q^{H}=A_{n} \cdots A_{1}$. Further unitary rotation matrix $Q$ will be estimated exclusively using training sequence based on OPML algorithm which already explained in Section 3.1, where Whitening matrix $W$ in Equation (2) is replaced by upper triangular matrix $R$. Finally estimated channel matrix is $\hat{H}=H \hat{Q}_{H}$.

\subsection{Joint Semi-Blind Channel and Data Estimation QR-NEW (Proposed Method-II)}

This novel method based on joint channel and data estimation using QR-decomposition algorithm. Let $X_{p}$ is pilot (training) and their corresponding output is $Y_{p}$, so initial channel estimation is calculated using pilot (training) data only using QR decomposition. Minimizing the norm square error function of Equation (4)

$$
\varepsilon=Y_{p}-X_{p} \hat{h}
$$

To avoid matrix inversion, we can directly apply QR decomposition to the error function and estimate initial channel using following steps

1) $\varepsilon=Y_{p}-X_{p} \hat{h}$ and if $\varepsilon=0 \Rightarrow Y_{p}=X_{p} \hat{h}$

2) Decompose $X_{p}$ into, hermitian matrix $Q_{p}$ and upper triangular matrix $R_{p}$ using $\mathrm{QR}$ decomposition algorithm.

$$
Y_{p}=X_{p} \hat{h}=Q_{p}\left[\begin{array}{c}
R_{p} \\
0
\end{array}\right] \hat{h}
$$

where $Q Q^{H}=I$ due to hermitian property multiply both sides by $Q^{H}$ therefore,

$$
\left[\begin{array}{c}
R_{p} \\
0
\end{array}\right] \hat{h}=Y_{p} Q_{p}^{H}
$$

Then $\hat{h}$ is estimated using back-substitution method.

From that $\hat{h}$ we can find estimated blind data $X_{b e s t}=\hat{h}^{H} Y_{b}$ where $Y_{b}$ is received output data. Now perform same procedure to minimize norm square error function

$$
\varepsilon=Y_{b}-X_{\text {best }} \hat{H} \text {, so } \varepsilon=0 \Rightarrow Y_{b}=X_{\text {best }} \hat{H}
$$

Decompose $X_{b e s t}$ into, hermitian matrix $Q_{b}$ and upper triangular matrix $R_{b}$ using $\mathrm{QR}$ decomposition algorithm 


$$
Y_{b}=Q_{b}\left[\begin{array}{c}
R_{b} \\
0
\end{array}\right] \hat{H}
$$

Now $Q_{b} Q_{b}^{H}=I$, multiplying both side, therefore

$$
\left[\begin{array}{c}
R_{b} \\
0
\end{array}\right] \hat{H}=Y_{b} Q_{b}^{H}
$$

Final channel estimation $\hat{H}$ is performed using back substitution method.

\subsection{Least Square (LS) Training Based Channel Estimation}

Received signal can be expressed in Equation (4). The channel estimation is to find a solution $\hat{H}$ for the equation $X \hat{H} \approx Y$. What LS mean is to minimize the Euclidean norm squared of the residual $A \hat{H}-Y$, that is,

$$
\begin{gathered}
\|X \hat{H}-Y\|^{2}=(X \hat{H}-Y)^{H}(X \hat{H}-Y) \\
(X \hat{H})^{H}(X \hat{H})-Y^{H} X \hat{H}-(X \hat{H})^{H} Y+Y^{H} Y
\end{gathered}
$$

Differentiating with respect to $H$ and setting equal to 0 yields

$$
2 X^{H} X \hat{H}-2 X^{H} Y=0 \rightarrow X^{H} X \hat{H}+X^{H} Y
$$

Finally LS algorithm

$$
\hat{H}_{\mathrm{LS}}=\left(X^{H} X\right)^{-1} X^{H} Y
$$

The term $\left(X^{T} X\right)^{-1} X^{H}$ is called pseudo-inverse of matrix $X$, denoted by $X^{\dagger}$.

\section{Simulation Results}

Extensive computer simulations have been conducted to demonstrate and compare the performance of Training based LS, WR based semi-blind channel estimation and proposed novel semi-blind channel estimation techniques for Rayleigh flat fading MIMO channels. We consider alamouti coded $2 \times 6$ (two transmitters and six receivers) MIMO systems with 100 blind data symbols among 20,000 pair transmitted symbols under 4-PSK modulation scheme using 4, 8 and 16 pilot symbols. Result shows in figures depicts that semi-blind channel estimation techniques have better BER performance than training based LS channel estimation technique further first novel technique with perfect $\mathrm{R}$ outperforms others.

\subsection{BER (Bit Error Rate)}

As a performance measure, BER is evaluated for training based LS channel estimation technique, WR based semi-blind channel estimation technique, proposed ma- trix triangularization based Householder QR decomposition semi-blind channel estimation (first technique) and proposed QR decomposition based joint semi-blind channel and data estimation technique (second technique) in a Rayleigh flat fading MIMO channel. Simulation results are related to three cases: MIMO $2 \times 6$ with 4 pilots, 8 pilots and 16 pilots respectively.

\subsubsection{MIMO $2 \times 6$ with 4 Pilots}

Figure 1 indicates the BER Vs SNR for above mentioned different channel estimation techniques using 4 pilot symbols and 100 blind symbols for 2 transmitter and 6 receiver antennas under 4 PSK modulation scheme. First novel technique with perfect $\mathrm{R}$ outperforms WR technique with $1 \mathrm{~dB}$ to $2 \mathrm{~dB}$ improvements. Both newly proposed techniques shows similar performance, with $0.4 \mathrm{~dB}$ to $0.5 \mathrm{~dB}$ performance losses compare to conventional WR technique. When SNR equals to $2 \mathrm{~dB}$, BER is 0.0320 for TBCE-LS, 0.0243 for QR-NEW (proposed technique-II), 0.0207 for WR and 0.0153 for HQROPML (proposed technique-I) with perfect R, further at $5 \mathrm{~dB}$ SNR, BER goes around $10^{-3}$.

\subsubsection{MIMO $2 \times 6$ with 8 Pilots}

Figure 2 indicates the BER Vs SNR for same analysis using 8 pilot symbols .Here when SNR equals to $2 \mathrm{~dB}$, BER is 0.0154 for TBCE-LS, 0.0127 for QR-NEW (proposed technique-II), 0.0115 for WR and 0.091 for HQR-OPML (proposed technique-I) with perfect R., further at $5 \mathrm{~dB}$ SNR, BER goes slightly greater than $10^{-4}$, So 1 to $1.5 \mathrm{~dB}$ performance improvements compared to 4 pilots scheme.

\subsubsection{MIMO $2 \times 6$ with 16 Pilots}

BER performance for 16 pilots demonstrated in Figure 3 shows further performance improvements compared to previous cases. Here at $2 \mathrm{~dB}$ SNR, BER is 0.0102 for TBCE-LS, 0.0100 for QR-NEW, 0.081 for WR and 0.042 for HQR-OPML with perfect R. At $5 \mathrm{~dB}$ SNR, BER is 0.0003 , which considered as best one. Further it shows almost $2 \mathrm{~dB}$ improvements compared to past cases.

\subsubsection{Complexity Comparisons}

Complexity analysis of channel estimation techniques have been taken for $\mathrm{m}$ receiver matrix and $\mathrm{n}$ transmitter MIMO channel matrix, Whitening Rotation based semi-blind channel estimation technique consists of two singular value decomposition (SVD) and two matrix multiplication operations. The SVD and multiplication process having total $\mathrm{O}\left(\mathrm{mn}^{2}\right)$ and $(\mathrm{mn})$ floating point operations respectively hence total complexity is $\mathrm{O}(2$ $\left.m n^{2}+2 m n\right)$. Proposed householder QR decomposition 


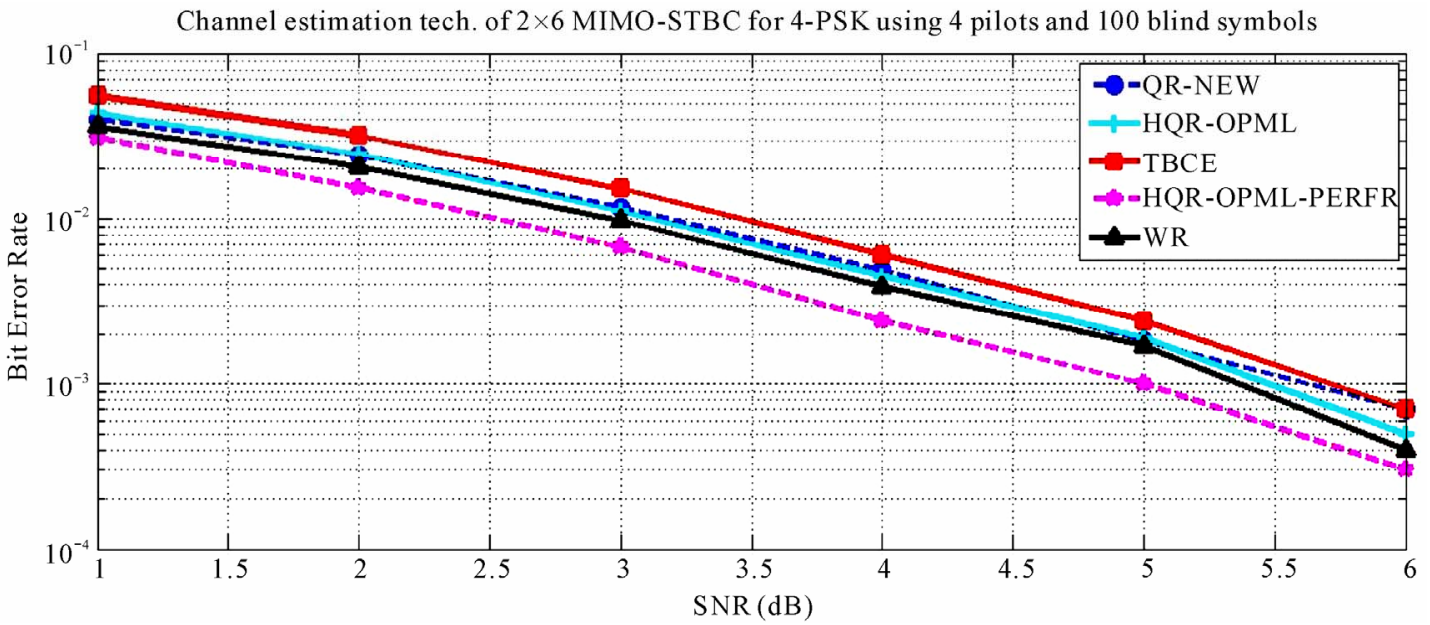

Figure 1. BER Vs SNR for diff. channel estimation techniques using 4 Training and 100 Blind symbols for 2 transmitter and 6 receiver antennas for 4 PSK modulation scheme.

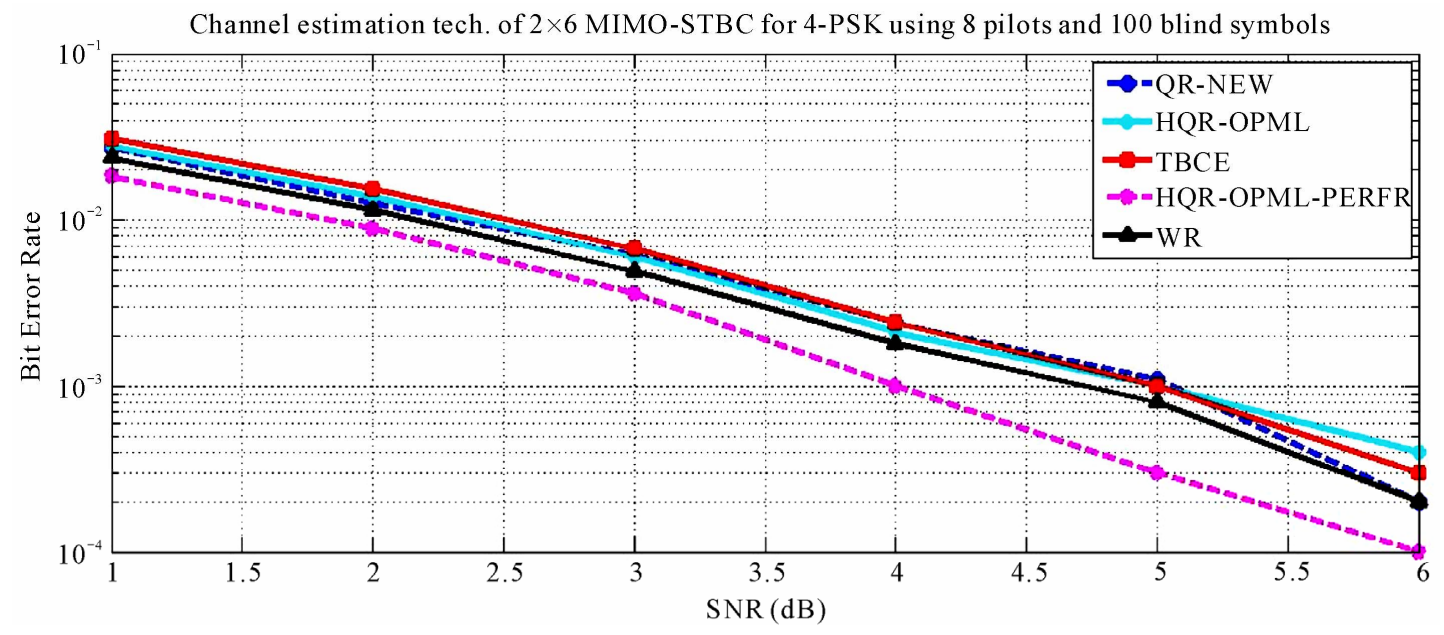

Figure 2. BER Vs SNR for diff. channel estimation techniques using 8 Training and 100 Blind symbols for 2 transmitter and 6 receiver antennas for 4 PSK modulation scheme.

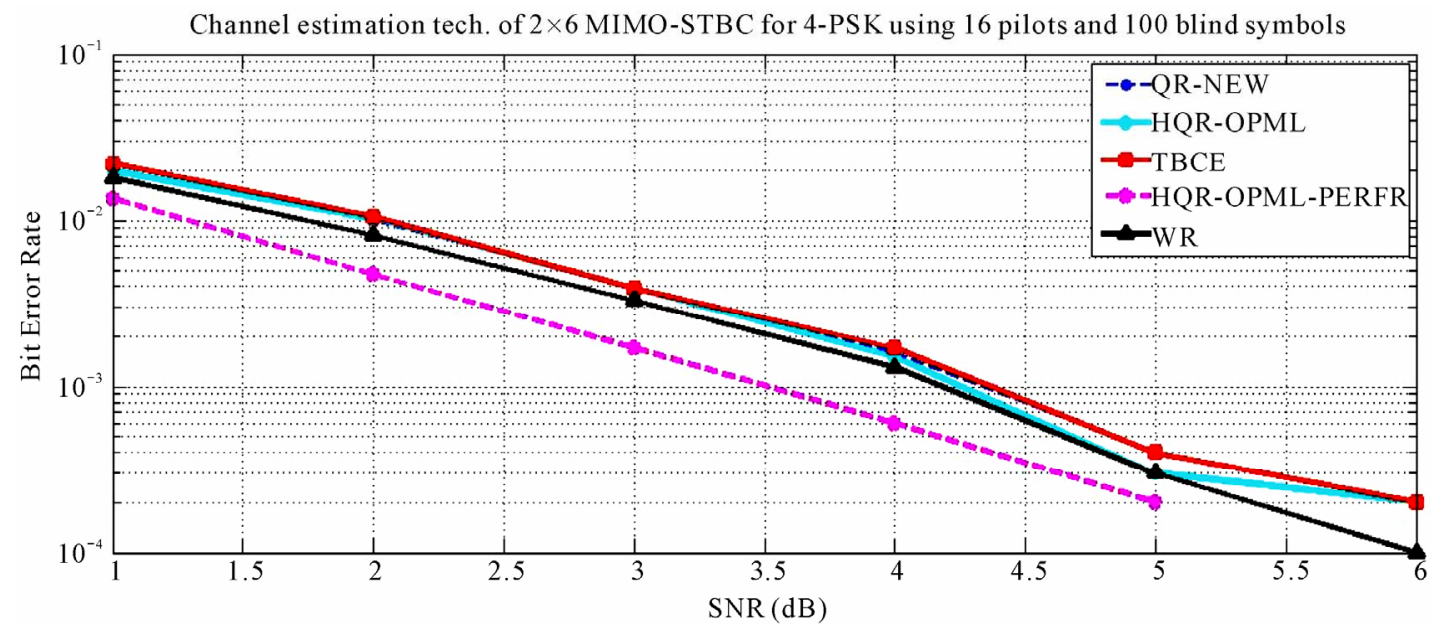

Figure 3. BER Vs SNR for diff. channel estimation techniques using 16 Training and 100 Blind symbols for 2 transmitter and 6 receiver antennas for 4 PSK modulation scheme. 
based first technique consists of householder reflection QR operation, which having $\mathrm{O}\left(\mathrm{n}^{3}\right)$ floating point operations, further its having one SVD and two multiplication process so total floating point operations are $\mathrm{O}\left(\mathrm{n}^{3}+\mathrm{mn}^{2}\right.$ $+2 \mathrm{mn}$ ). Second novel technique having two QR decomposition process with three multiplication process, hence total operations related to this technique are $\mathrm{O}\left(2 \mathrm{n}^{3}+\right.$ $3 \mathrm{mn}$ ). Finally this analysis depicts that novel techniques having less complexity compare to Whitening Rotation based semi-blind channel estimation technique.

\section{Conclusions}

The work investigated for Householder based QR-OPML and joint channel and data estimation based QR-NEW semi-blind channel estimation techniques for Rayleigh flat fading MIMO channel using two transmitter and six receiver antennas combinations and various pilot symbols. The simulations result shows that BER performance improves as number pilot symbols increase. Finally those techniques compare with Whitening rotation (WR) based semi-blind channel estimation technique and observed House holder QR-OPML based first novel technique with perfect $\mathrm{R}$ outperforms WR based semi-blind technique with low complexity.

\section{References}

[1] M. Kiessling, J. Speidel and Y. Chen, "MIMO Channel Estimation in Correlated Fading Environments," Proceeding of 58th IEEE Vehicular Technology Conference (VTC'03), Orlando, 8 October 2003, pp. 1187-1191.

[2] G. Xie, X. Fang, A. Yang and Y. Liu, "Channel Estimation with Pilot Symbol and Spatial Correlation Information," Proceeding of IEEE International Symposium on Communication and Information Technologies (ISCIT'07), Sydney, 16-19 October 2007, pp. 1003-1006.

[3] S. M. Kay, "Fundamentals of Statistical Signal Processing: Estimation Theory," Prentice Hall, Upper Saddle River, 1993.

[4] G. K. Krishnan and V. U. Reddy, "MIMO Communication-Motivation and a Practical Realization," IETE Technical Review, Vol. 24, No. 4, 2007, pp. 203-213.

[5] T. Wo, P. A. Hoeher, A. Scherb and K. D. Kammeyer, "Performance Analysis of Maximum-Likelihood Semi Blind Estimation of MIMO Channel," Proceedings of 63rd IEEE vehicular Technology Conference (VCT), Melbourne, 7-10 May 2006, pp. 1738-1742.

[6] S. Chen, X. C. Yang, L. Chen and L. hanzo, "Blind Joint Maximum Likelihood Channel Estimation and Data Detection for SIMO System," International Journal for Computer, Vol. 4, No. 1, 2007, pp. 47-51.

[7] K. Sabri, M. El Badaoui, F. Guillet, A. Adib and D. Aboutajdine, "A Frequency Domain Based Apporch for Blind MIMO System Identification Using Second Order
Cyclic Statistics," Elsevier Signal Processing, Vol. 89, No. 1, 2009, pp. 77-86. doi:10.1016/j.sigpro.2008.07.011

[8] I. M. Panahi and K. Venket, "Blind Identification of Multi-Channel System with Single Input and Unknown Order," Elsevier Signal Processing, Vol. 89, No. 7, 2009, pp. 1288-1310. doi:10.1016/j.sigpro.2008.12.007

[9] M. Abuthinien, S. Chen, A. Wolfgang and L. Hanzo, "Jont Maximum Likelihood Channel Estimation and Data Detection for MIMO System," In Proceeding of IEEE International Conference on Communication (ICC'07), Glasgow, 24-28 July 2007, pp. 5354-5358.

[10] M. A. Khalighi and S. Bourennane, "Semiblind SingleCarrier MIMO Channel Estimation Using Overlay Pilots," IEEE Transactions on Vehicular Technology, Vol. 57, No. 3, May 2008, pp. 1951-1956. doi:10.1109/TVT.2007.907080

[11] B. Chen and A. P. Petropulu, "Frequency Domain Blind Mimo System Identification Based on Second and Higher Order Statistics," IEEE Transactions on Signal Processing, Vol. 49, No. 8, 2001, pp. 1677-1688. doi:10.1109/78.934137

[12] E. De Carvalho and D. T. M. Slock, "Asymptotic Performance of ML Methods for Semi-Blind Channel Estimation," 31st Asilomar Conference, Pacific Grove, 2-5 November 1998, pp. 1624-1628.

[13] A. Medles, D. T. M. Slock and E. D. Carvalho, "Linear Prediction Based Semi-Blind Estimation of MIMO FIR Channels," 3rd IEEE SPAWC, Taiwan, 20-23 March 2001, pp. 58-61.

[14] A. K. Jagannatham and B. D. Rao, "A Semi-Blind Technique for MIMO Channel Matrix Estimation," In Proceedings of IEEE Workshop on Signal Processing Advances in Wireless Communications (SPAWC 2003), Rome, 15-18 June 2003, pp. 304-308.

[15] X. Liu, F. Wang and M. E. Bialkowski "Investigation into a Whitening-Rotation-Based Semi-Blind MIMO Channel Estimation for Correlated," International Conference on Signal Processing and Communication Systems, ICSPCS, Gold Coast, 15-17 December 2008, pp. 1-4.

[16] Q. W. Zhang, W.-P. Zhu and Q. M. Meng, "Whitening-Rotation-Based Semi-Blind Estimation of MIMO FIR Channels," International Conference on Wireless Communications \& Signal Processing, WCSP, Nanjing, 13-15 November 2009, pp. 1-4.

[17] F. Wan, W.-P. Zhu and M. N. S. Swamy, "Perturbation Analysis of Whitening-Rotation-Based Semi-Blind MIMO Channel Estimation," IEEE International Midwest Symposium on Circuits and Systems, MWSCAS'09, Cancun, 2-5 August 2009, pp. 240-243.

[18] J. G. Proakis, "Digital Communications," McGraw-Hill Higher Education, New York, 2001.

[19] D. Pal, "Fractionally Spaced Semi-Blind Equalization of Wireless Channels," The 26th Asilomar Conference, Vol. 2, 1992, pp. 642-645.

[20] F. Wan, W.-P. Zhu and M. N. S. Swamy, "A Semi-Blind Channel Estimation Approach for MIMO-OFDM Systems," IEEE Transactions on Signal Processing, Vol. 56, 
No. 7, 2008, pp. 2821-2834. doi:10.1109/TSP.2008.917354

[21] A. K. Jagannatham and B. D. Rao, "Whitening-Rotation-Based Semiblind MIMO Channel Estimation," IEEE Transactions on Signal Processing, Vol. 54, No. 3, 2006, pp. 861-869. doi:10.1109/TSP.2005.862908

[22] T. Wo and P. A. Hoeher, "Semi-Blind Channel Estimation for Frequency Selective MIMO System," IST Mobile
\& Wireless Communications Summit, Dresden, 19-23 June 2005.

[23] T. Cui and C. Tellambura, "Semiblind Channel Estimation and Data Detection for OFDM System with Optimal Pilot Design," IEEE Transactions on Communications, Vol. 55, No. 5, May 2007, pp. 1053-1062.

doi:10.1109/TCOMM.2007.895985 